OPEN ACCESS

Edited by:

Alessio Fini,

University of Florence, Italy

Reviewed by:

Abu Hena Mostafa Kamal,

University of Texas at Arlington, USA

Josselin Noirel,

Conservatoire National des Arts et

Métiers, France

*Correspondence:

Liwang Liu

nauliulw@njau.edu.cn

${ }^{\dagger}$ These authors have contributed equally to this work.

Specialty section:

This article was submitted to Functional Plant Ecology,

a section of the journal

Frontiers in Plant Science

Received: 12 August 2016 Accepted: 28 November 2016 Published: 15 December 2016

Citation:

Wang $Y, X u L$, Tang $M$, Jiang $H$, Chen W, Zhang W, Wang R and Liu L (2016) Functional and Integrative Analysis of the Proteomic Profile of

Radish Root under Pb Exposure. Front. Plant Sci. 7:1871. doi: 10.3389/fp/s.2016.01871

\section{Functional and Integrative Analysis of the Proteomic Profile of Radish Root under $\mathrm{Pb}$ Exposure}

\author{
Yan Wang ${ }^{\dagger}$, Liang Xu ${ }^{\dagger}$, Mingjia Tang, Haiyan Jiang, Wei Chen, Wei Zhang, Ronghua Wang \\ and Liwang Liu *
}

National Key Laboratory of Crop Genetics and Germplasm Enhancement, College of Horticulture, Nanjing Agricultural University, Nanjing, China

Lead $(\mathrm{Pb})$ is one of the most abundant heavy metal $(\mathrm{HM})$ pollutants, which can penetrate the plant through the root and then enter the food chain causing potential health risks for human beings. Radish is an important root vegetable crop worldwide. To investigate the mechanism underlying plant response to $\mathrm{Pb}$ stress in radish, the protein profile changes of radish roots respectively upon $\mathrm{Pb}\left(\mathrm{NO}_{3}\right)_{2}$ at $500 \mathrm{mg} \mathrm{L}^{-1}(\mathrm{~Pb} 500)$ and $1000 \mathrm{mg}$ $\mathrm{L}^{-1}$ (Pb1000), were comprehensively analyzed using iTRAQ (Isobaric Tag for Relative and Absolute Quantification). A total of 3898 protein species were successfully detected and 2141 were quantified. Among them, a subset of 721 protein species were differentially accumulated upon at least one $\mathrm{Pb}$ treatment, and 135 ones showed significantly abundance changes under both two Pb-stressed conditions. Many critical protein species related to protein translation, processing, and degradation, reactive oxygen species (ROS) scavenging, photosynthesis, and respiration and carbon metabolism were successfully identified. Gene Ontology (GO) and pathway enrichment analysis of the 135 differential abundance protein species (DAPS) revealed that the overrepresented GO terms included "cell wall," "apoplast," "response to metal ion," "vacuole," and "peroxidase activity," and the critical enriched pathways were involved in "citric acid (TCA) cycle and respiratory electron transport," "pyruvate metabolism," "phenylalanine metabolism," "phenylpropanoid biosynthesis," and "carbon metabolism." Furthermore, the integrative analysis of transcriptomic, miRNA, degradome, metabolomics and proteomic data provided a strengthened understanding of radish response to $\mathrm{Pb}$ stress at multiple levels. Under Pb stress, many key enzymes (i.e., ATP citrate lyase, Isocitrate dehydrogenase, fumarate hydratase and malate dehydrogenase) involved in the glycolysis and TCA cycle were severely affected, which ultimately cause alteration of some metabolites including glucose, citrate and malate. Meanwhile, a series of other defense responses including ascorbate (ASA)-glutathione (GSH) cycle for ROS scavenging and Pb-defense protein species (glutaredoxin, aldose 1-epimerase malate dehydrogenase and thioredoxin), were triggered to cope with $\mathrm{Pb}$-induced injuries. These results would be helpful for further dissecting molecular mechanism underlying plant response to $\mathrm{HM}$ stresses, and facilitate effective management of $\mathrm{HM}$ contamination in vegetable crops by genetic manipulation.

Keywords: proteomics, radish, iTRAQ, heavy metal, lead stress 


\section{INTRODUCTION}

Heavy metal (HM) contamination from natural, agricultural, and industrial sources has become a worldwide public health concern, which could seriously deteriorate the environment and cause adverse impacts on human health through the food chain (Pourrut et al., 2013; Singh et al., 2016). Lead (Pb) is one of the most abundant HM pollutants with no physiological function, mostly penetrating the plant through the roots and accumulating in different parts (Gupta et al., 2013; Pourrut et al., 2013). Pb can influence various morphological, physiological and biochemical processes leading to a restriction of plant growth, inducing membrane cell damages and, ultimately, to cell death (Sharma and Dubey, 2005; Gupta et al., 2011). Accordingly, plants also have developed diverse defense mechanisms to prevent the toxic effect of heavy metals including generating signal sensing and transduction proteins, compartmenting into the vacuoles and induction of higher levels of metal chelates like a protein complex, organic and inorganic complexes (Gupta et al., 2011, 2013; Thapa et al., 2012).

Radish (Raphanus sativus L.), a major member of the Brassicaceae family, is an important annual or biennial root vegetable crop worldwide (Wang and $\mathrm{He}, 2005$ ). Because the root is considered as the vulnerable part which is easily affected by HM (Wang et al., 2015a), it has become of vital importance to investigate the HM-response mechanisms and explore the molecular regulatory network of tolerance and homeostasis in radish. The identification of the HM-responsive genes or proteins is a fundamental step in understanding the molecular mechanism underlying response to HM stress (Ahsan et al., 2009). In our previous study, NGS-based transcriptome, miRNA and degradome analysis were employed to investigate the expression patterns of genes and miRNAs in radish exposure to $\mathrm{Pb}$ stress. A lot of Pb-responsive transcripts, miRNA and its targets were detected, which were predominately involved in stressrelated signal sensing and transduction, specific metal uptake and homeostasis, glutathione metabolism-related processes and carbohydrate metabolism-related pathways (Wang et al., 2013, 2015b).

Although transcriptomics provides a useful tool for unraveling gene expression networks at the mRNA level which enhanced our understanding the response of radish under $\mathrm{Pb}$ stress, proteomics can offer a new platform for investigating complex biological functions involving large numbers of proteins and provide further insight into posttranscriptional modifications thereby complementing genomics analysis (Ralhan et al., 2008; Ahsan et al., 2009; Wang et al., 2014). In the past two decades, classical two-dimensional electrophoresis (2-DE) technology has been widely employed for protein identification and analysis. However, there were some limitations in its applications such as poor reproducibility, weak sensitivity and low automation (Sazuka et al., 2004). Isobaric tags for relative and absolute quantification (iTRAQ) is a robust mass spectrometry of protein quantitative technology, which can perform relative and absolute quantification in up to eight samples in parallel (Bindschedler and Cramer, 2011; Glibert et al., 2014). Recently, iTRAQ has been widely used for large-scale quantitative plant proteomic studies in exploration of various metabolic processes at the post-transcriptional level (Kambiranda et al., 2013; MartínezEsteso et al., 2013) in response to various stresses (Yang et al., 2014; Li et al., 2015; Fu et al., 2016). Additionally, iTRAQbased proteomics has been proven as a powerful method for unraveling the molecular regulatory networks involved in interactions between heavy metals and plant species, and a set of HM-responsive candidate proteins have been successfully identified. Alvarez et al. (2009) reported that exposure of Brassica juncea roots to cadmium (Cd) could activated several protein species involved in sulfur assimilation, redox homeostasis and xenobiotic detoxification, and depressed multiple proteins involved in protein synthesis and processing by two quantitative proteomics approaches including fluorescence two-dimensional differential gel electrophoresis (DIGE) and iTRAQ technology. More recently, the protein abundance changes in rice roots in response to Aluminum (Al) at an early phase were conducted with iTRAQ, and a total of 700 distinct protein species with $>95 \%$ confidence were identified and 106 protein species were differentially accumulated upon Al toxicity in sensitive and tolerant cultivars (Wang et al., 2014). However, investigation of the dynamically protein abundance changes in response to $\mathrm{Pb}$ stress in radish has not been reported.

In this study, an iTRAQ-based quantitative proteomics approach was firstly employed to detect the effects of $\mathrm{Pb}$ responses at the protein abundance levels in radish roots. The differential abundance protein species (DAPS) involved in $\mathrm{Pb}$ response of radish were quantified, and the enriched networks for regulating $\mathrm{Pb}$ stress at the protein level were acquired. Furthermore, to deeply reveal the integrative molecular network of radish plant response to $\mathrm{Pb}$ stress, the proteomic data were integrated with our previous transcriptomic, miRNA, degradome and metabolomic data, which provided a more global view of the molecular and cellular changes elicited by $\mathrm{Pb}$ stress in radish. This work would provide valuable information for further functional analyses of the critical $\mathrm{Pb}$-responsive protein species in radish, which will be helpful for effectively facilitating the management of $\mathrm{Pb}$ and other $\mathrm{HM}$ contaminations in vegetable crops by genetic manipulation.

\section{MATERIALS AND METHODS}

\section{Plant Material}

The variety of high- $\mathrm{Pb}$-accumulation "NAU-RG" was selected for exploring the molecular regulation mechanisms in radish roots responding to the $\mathrm{Pb}$ stress. This genotype is an advanced inbred line with a medium size root in globular shape, white skin and flesh. According to the related evaluation methods and previous studies, different concentrations of $\mathrm{Pb}\left(\mathrm{NO}_{3}\right)_{2}(100,200,400,500$, 1000 , and $1500 \mathrm{mg} \cdot \mathrm{L}^{-1}$ ) were set to investigate the changes of visible physiological symptoms with different temporal durations in the preliminary experiment. Interestingly, no special obvious morphologic differences were found among individuals when exposed to low dose of heavy metal treatment, while the plants were seriously hampered and grew abnormally when exposed to $1500 \mathrm{mg} \cdot \mathrm{L}^{-1} \mathrm{~Pb}\left(\mathrm{NO}_{3}\right)_{2}$. Therefore, the concentrations of $\mathrm{Pb}$ 
$\left(\mathrm{NO}_{3}\right)_{2}$ at $500 \mathrm{mg} \cdot \mathrm{L}^{-1}(\mathrm{~Pb} 500)$ and $1000 \mathrm{mg} \cdot \mathrm{L}^{-1}(\mathrm{~Pb} 1000)$ were selected for the comparative proteomic analysis. Additionally, a control group was defined using non-treated seedlings (CK). The growth conditions of radish plants were conducted according to the reported descriptions (Wang et al., 2013). Plants were collected after $72 \mathrm{~h}$ with three different concentration treatments including an untreated control (CK) and two Pb-stressed conditions ( $\mathrm{Pb} 500$ and $\mathrm{Pb} 1000)$, based on the previous reported study (Wang et al., 2013). Each treatment consisted of three biological replicates. Equal amount of radish taproot samples $(2.5 \mathrm{~g})$ from three randomly selected individual plants of each replicate were pooled and cut into small pieces, which were rapidly frozen in liquid nitrogen and stored at $-80^{\circ} \mathrm{C}$ for protein extraction.

\section{Protein Extraction}

Total protein was extracted from the radish taproots using a phenol (Phe) extraction procedure according to the reported methods with some modifications (Saravanan and Rose, 2004; Yang et al., 2013). In brief, about $2.5 \mathrm{~g}$ frozen taproots were finely powdered in liquid nitrogen adding 0.5 polyvinylpolypyrrolidone (PVPP) and then suspended in $20 \mathrm{~mL}$ of precooled extraction buffer containing $500 \mathrm{mM}$ Tris- $\mathrm{HCl} \mathrm{pH}$ 7.5, $50 \mathrm{mM}$ ethylenediaminetetraacetic acid (EDTA), $100 \mathrm{mM}$ $\mathrm{KCl}, 2 \mathrm{mM}$ dithiothreitol (DTT), $2 \mathrm{mM}$ phenylmethylsulfonyl (PMSF) and 30\% sucrose (w/v). The mixture was extensively homogenized on ice for $2 \mathrm{~min}$, and then an equal volume of Tris- $\mathrm{HCl} \mathrm{pH}$ 7.5-saturated Phe was added. The mixture was thoroughly vortexed and proteins were collected by centrifuging at $12,000 \mathrm{~g}$ for $15 \mathrm{~min}$ at $4^{\circ} \mathrm{C}$. The upper Phe phase was removed and re-extracted two or three times with extraction buffer. Proteins were precipitated from the final Phe phase with five volumes of saturated ammonium acetate in methanol at $-20^{\circ} \mathrm{C}$ overnight. After centrifuging at $12,000 \mathrm{~g}$ for $15 \mathrm{~min}$ at $4^{\circ} \mathrm{C}$, the pellets were washed twice with $20 \mathrm{~mL}$ of $0.1 \mathrm{~mol} \cdot \mathrm{L}^{-1}$ cooled acetone, and then dried by lyophilization and finally stored at $-80^{\circ} \mathrm{C}$ until use. The protein was quantified by a Bradford protein assay kit (Sangon Biotech, China), and an equal amount of protein from three replicates of each treatment $(\mathrm{CK}, \mathrm{Pb} 500$, and $\mathrm{Pb} 1000)$ was respectively pooled for iTRAQ analysis.

\section{iTRAQ Labeling and Strong Cation Exchange (SCX) Chromatography}

The protein samples were dissolved in $100 \mathrm{mM}$ triethylammonium bicarbonate (TEAB, $\mathrm{pH}$ 8.5) containing $1 \%$ SDS (w/v), reduced with $10 \mathrm{mM} \mathrm{DTT}$ at $56^{\circ} \mathrm{C}$ for $1 \mathrm{~h}$, and followed by alkylation with $55 \mathrm{mM}$ iodoacetamide (IAM) for $45 \mathrm{~min}$ at room temperature in the dark. Trypsin was then added to a final enzyme/substrate ratio of 1:20 (w/w) for protein digestion, which was incubated at $37^{\circ} \mathrm{C}$ for $12 \mathrm{~h}$. The resulting tryptic peptides were vacuum-concentrated and then labeled with iTRAQ reagents (Applied Biosysterms, USA) according to manufacturer's instruction. The control sample (CK) was labeled with 118 iTRAQ reagent, and $\mathrm{Pb}$-stressed samples treated with 500 and $1000 \mathrm{mg} \cdot \mathrm{L}^{-1} \mathrm{~Pb}\left(\mathrm{NO}_{3}\right)_{2}$ were labeled with 119 and 114 , respectively. The labeling reactions were incubated for
$2 \mathrm{~h}$ at room temperature. Subsequently, all the peptides from three groups were combined and further fractionated using SCX chromatography on a Ultremex SCX column $(250 \times 4.6 \mathrm{~mm}$, $5 \mu \mathrm{m}$ particle size, $200 \AA$ pore size, Phenomenex, USA) by high performance liquid chromatography (HPLC) system (Shimadzu LC-20AB, Japan). The HPLC gradient consisted of buffer A (25 $\mathrm{mM} \mathrm{NaH}_{2} \mathrm{PO} 4,25 \% \mathrm{ACN}, \mathrm{pH} 2.7$ ) for $10 \mathrm{~min}, 5-35 \%$ buffer B (25 mM NaH $\left.\mathrm{mO}_{4}, 25 \% \mathrm{ACN}, 1 \mathrm{M} \mathrm{KCL}, \mathrm{pH} 2.7\right)$ for $11 \mathrm{~min}$ and $35-80 \%$ buffer $B$ for $1 \mathrm{~min}$, which were eluted at a flow rate of $1 \mathrm{~mL} \cdot \mathrm{min}^{-1}$. The chromatograms were recorded at $214 \mathrm{~nm}$ and 20 constituents were obtained. The collected fractions were desalted with StrataX (Phenomenex, USA) and concentrated to dryness using a vacuum centrifuge.

\section{Liquid Chromatography Coupled with Tandem Mass Spectrometry (LC-MS/MS)}

The mass spectroscopy analysis was performed using an ABSCIE X TripleTOF 5600 mass spectrometer (AB SCIE $\mathrm{X}$, Framingham, MA, USA), coupled with an online flow HPLC nanoAcquity system (Waters, USA), which comprised two parts of columns including Symmetry C18 column $(5 \mu \mathrm{m}$ particles, $180 \mathrm{um} \times 20 \mathrm{~mm})$ and BEH130 C18 column specification $(1.7 \mu \mathrm{m}$ particles, $100 \mu \mathrm{m} \times 100 \mathrm{~mm})$. Symmetry C18 column was used for adsorption and desalination of peptides, while BEH130 for the separation of peptides. The correction liquid (Thermo Fisher Scientific) was respectively added to liquid A (water:acetonitrile:formic acid = 98:2:0.1) and $\mathrm{B}$ (water:acetonitrile:formic acid $=2: 98: 0.1$ ) with a certain proportion. A $2.25 \mu \mathrm{g}$ aliquot of supernatant $(9 \mu \mathrm{l})$ was transferred, and then the samples were eluted with liquid A at $2 \mu \mathrm{l} \cdot \mathrm{min}^{-1}$ for $15 \mathrm{~min}$ for the purpose of adsorption and desalination of peptides. After that, the mobile phase which contain $5 \%$ liquid $\mathrm{B}$ was used to elute the supernatant $(300$ $\left.\mathrm{nl} \cdot \mathrm{min}^{-1}, 1 \mathrm{~min}\right)$. Next, the concentration of liquid B was raised from $5 \%$ to $35 \%$ in $40 \mathrm{~min}$, from $35 \%$ to $80 \%$ in 5 min using linear gradients, and then the samples were eluted for 5 min with $80 \%$ liquid $\mathrm{B}$. The separation was performed at a constant flow rate of $20 \mu \mathrm{l} \cdot \mathrm{min}^{-1}$.

\section{Data Analysis and Protein Identification}

Raw data files were converted into MGF files using Proteome Discoverer 1.2. Proteins identification were performed by using Mascot search engine (Matrix Science, London, UK; version 2.3.02) against our sequenced radish root transcriptome database (SRX316199 and http://www.ncbi.nlm.nih.gov/sra/; Wang et al., 2013). The search parameters were set according to the published studies (Qiao et al., 2012; Yang et al., 2013; Wang et al., 2014). For protein identification, a mass tolerance of $0.05 \mathrm{Da}$ was permitted for intact peptide masses and $0.1 \mathrm{Da}$ for fragmented ions, with allowance for one missed cleavages in the trypsin digests. The methionine oxidation $(\mathrm{M})$, protein $\mathrm{N}$-terminal acetylation, deamidation $(\mathrm{N}, \mathrm{Q})$, and iTRAQ $(\mathrm{Y})$ were selected as variable modifications, and carbamidomethyl modification of cysteines (C), iTRAQ (Nterminal), iTRAQ (K) were as fixed modifications. The search results were passed through additional filters before exporting the data. For protein identification, the filters were set as follows: significance threshold $P, 0.05$ (with 95\% confidence) 
and ion score or expected cutoff $<0.05$ (with $95 \%$ confidence). For protein quantitation, it was required that a protein contains at least two unique spectra. The quantitative protein ratios were weighted and normalized by the median ratio in Mascot. The DAPS were defined: Showing a fold-change of greater than 1.2 or less than 0.83 using a greater statistically significant value $P<0.05$.

\section{Functional Analysis}

The online software BLAST2GO (http://www.blast2go.com/ b2ghome) was used to automatically assign protein description and obtain annotations from homologous sequences of public databases (Conesa et al., 2005; Conesa and Götz, 2008). A metabolic pathway analysis was undertaken based on the Kyoto Encyclopedia of Genes and Genomes (KEGG) pathway database. The Gene Ontology (GO) and metabolic pathway enrichment analysis of the DAPS were conducted through two publically available tools (webservers), namely, DAVID 6.7 and KOBAS 2.0. Furthermore, an integrated expression analysis of $\mathrm{Pb}$-responsive miRNAs, mRNAs, metabolites and proteins were conducted based on our previous studies (Wang et al., 2013, 2015a,b). The same gene annotations were used to link expression levels across the four technologies.

\section{RESULTS}

\section{Protein Identification and Quantification of Radish Root in Response to Pb Stress}

A total of 241,107 spectra were obtained from the iTRAQ LC-MS/MS proteomic analysis of these three group samples including an untreated control (CK), Pb500 and Pb1000. After data filtering to eliminate low-scoring spectra, a total of 17,579 unique spectra were matched to 10,896 peptides representing 9725 unique peptides. The criteria that the peptides with $\geq$ distinct unique peptide were used for protein identification, and a total of 3898 protein species were successfully detected at a $95 \%$ confidence limit. The relative molecular mass distribution of the identified protein species showed that the most abundant sequences ranged from 10 to $80 \mathrm{kDa}$.

Only the proteins with at least two unique identified peptides were retained for further quantification analysis. In total, 2141 protein species were permitted the quantification of their abundance. Among them, 345 protein species were identified as differentially accumulated between $\mathrm{Pb} 500$ and $\mathrm{CK}$ groups including 177 (51\%) up-accumulated, and 168 (49\%) downaccumulated (Table S1). Among the 509 DAPS responding to the Pb1000 treatment, 48\% (244) showed increased abundance, while 52\% (265) showed decreased (Table S2). The proportion of common DAPS were calculated in response to the two $\mathrm{Pb}$ stress conditions. In total, 721 species were differentially accumulated under Pb treatments with 19\% (135) common DAPS in both $\mathrm{Pb}$ treatments and $81 \%$ (586) distinct DAPS, among which 211 unique to $\mathrm{Pb} 500$-stress and 375 unique to $\mathrm{Pb} 1000$-stress (Figure 1).

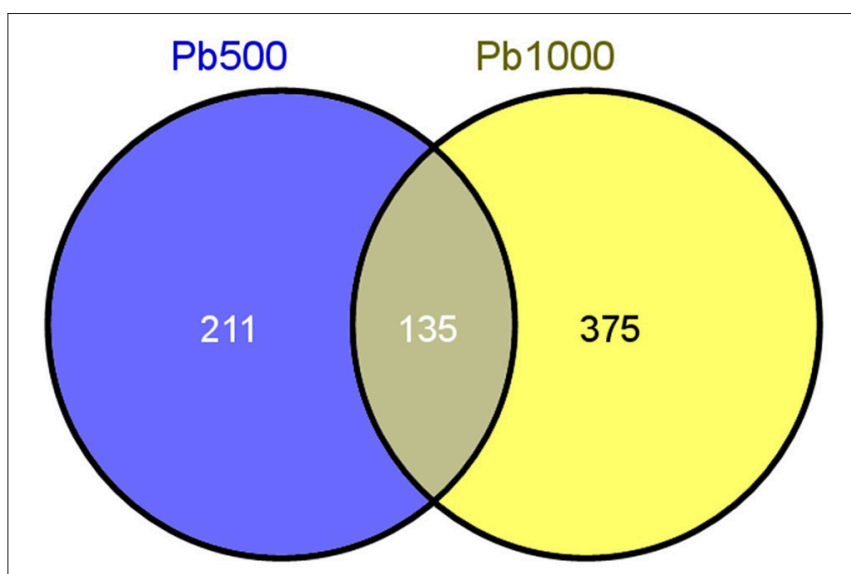

FIGURE 1 | The Venn diagram of differential abundance protein species (DAPS) involved in radish root under $\mathrm{Pb} 500$ and $\mathrm{Pb} 1000$ exposure.

\section{Functional Classification and Enrichment Analysis of the DAPS}

All of the 721 non-redundant DAPS sequences were functionally annotated using BLAST2GO (Conesa et al., 2005; Conesa and Götz, 2008). GO terms were assigned to query sequences and cataloged groups were produced basing on biological processes, molecular functions and cellular components. In total, 709 DAPS were assigned with $1687 \mathrm{GO}$ terms and could be classified into 43 functional groups at the second level 2 (Figure 2). Among them, biological processes represented the largest category, containing 22 groups with metabolic (78.0\%) and cellular process $(75.3 \%)$ as the two most frequent terms. Within the molecular function category, the predominant groups were assigned to catalytic activity (56.7\%) and binding (55.1\%). For cellular components, all the DAPS were mostly located in cell (84.2\%) and organelle $(67.7 \%)$.

To systematically understand its biological functions in terms of networks, the 721 DAPS were mapped into the KEGG pathway database. A total of 75 pathways were assigned, which were largely involved in carbohydrate metabolism (such as starch and sucrose metabolism, pyruvate metabolism, glycolysis/gluconeogenesis, pentose phosphate pathway, and amino sugar and nucleotide sugar metabolism), amino acid metabolism (phenylalanine metabolism, alanine, aspartate, and glutamate metabolism, cysteine and methionine metabolism and arginine and proline metabolism) and energy metabolism (carbon fixation in photosynthetic organisms, carbon fixation pathways in prokaryotes, methane metabolism, oxidative phosphorylation and sulfur metabolism) (Table S3). The pathways with the DAPS number larger than five were shown in Figure 3.

Furthermore, through functional enrichment analysis of the 135 common DAPS (Table 1), it was shown that "cell wall" (GO: 0005618), “apoplast” (GO: 0048046), "response to metal ion" (GO: 0010038), "vacuole" (GO: 0005773), and "peroxidase 


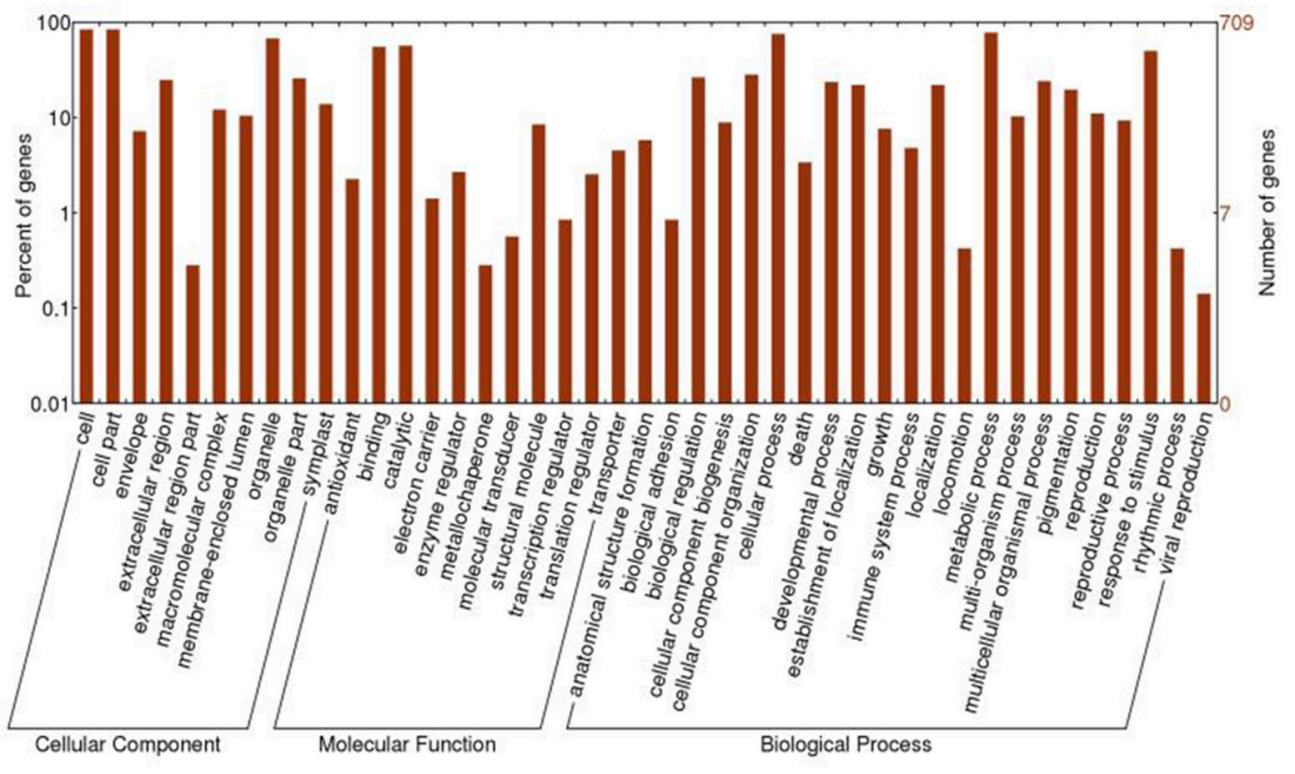

FIGURE 2 | GO classification of the DAPS of radish root in response to $\mathrm{Pb}$ stress.

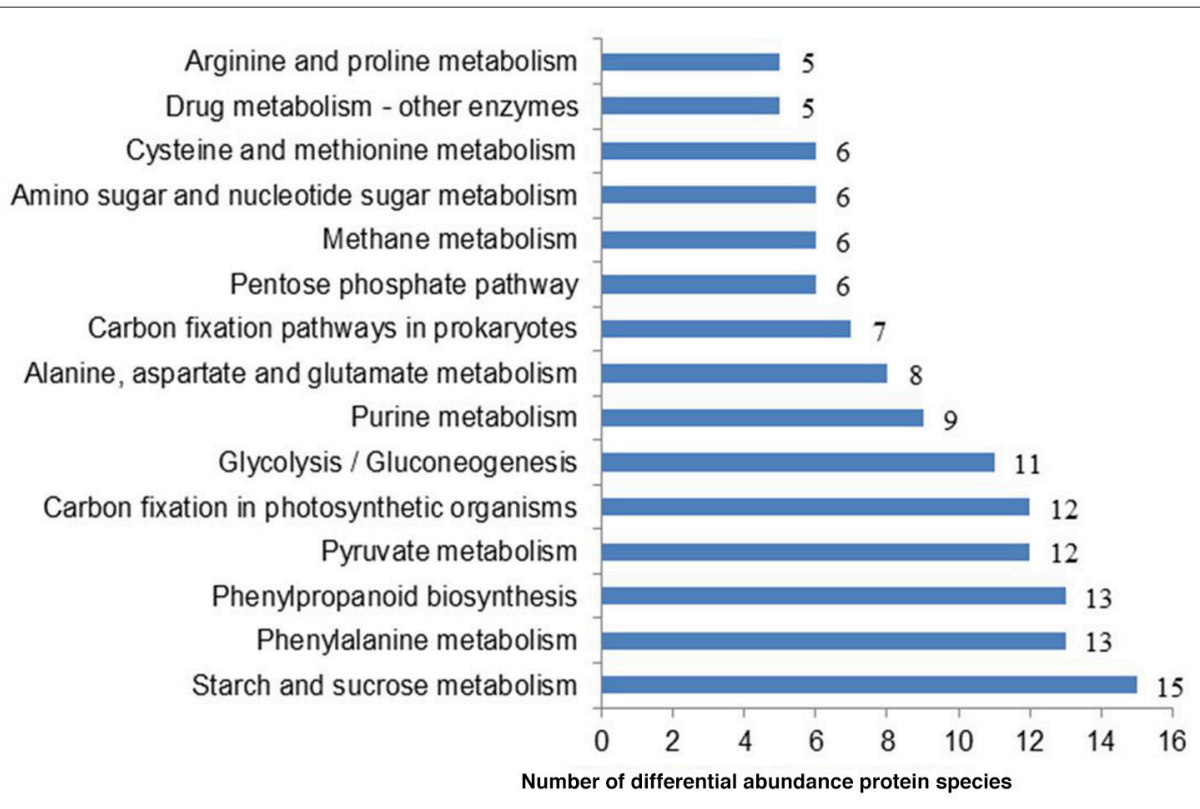

FIGURE 3 | The representative pathways of the DAPS involved in radish root response to $\mathrm{Pb}$ exposure.

activity" (GO: 0004601) were the most overrepresented GO terms (Table 2). KOBAS 2.0 webserver was employed to identify statistically enriched pathways from pathway databases involved in KEGG, BioCyc, Reactome, Pathway Interaction and Panther. The critical enriched pathways included "citric acid (TCA) cycle and respiratory electron transport," "pyruvate metabolism," "phenylalanine metabolism," "phenylpropanoid biosynthesis," and "carbon metabolism" (Table 3).

\section{Characterization of the Critical $\mathrm{Pb}$ Stress-Responsive Proteins in Radish}

Protein modification, the balance between synthesis and degradation, is a critical form of regulation that is coordinated to achieve a unified cellular under the stress of environmental stimuli (Hinkson and Elias, 2011). In this study, 27 protein species implicated in protein translation, processing and degradation were identified to be co-differentially accumulated 
TABLE 1 | The common DAPS involved in radish root under $\mathrm{Pb500}$ and Pb1000 exposure.

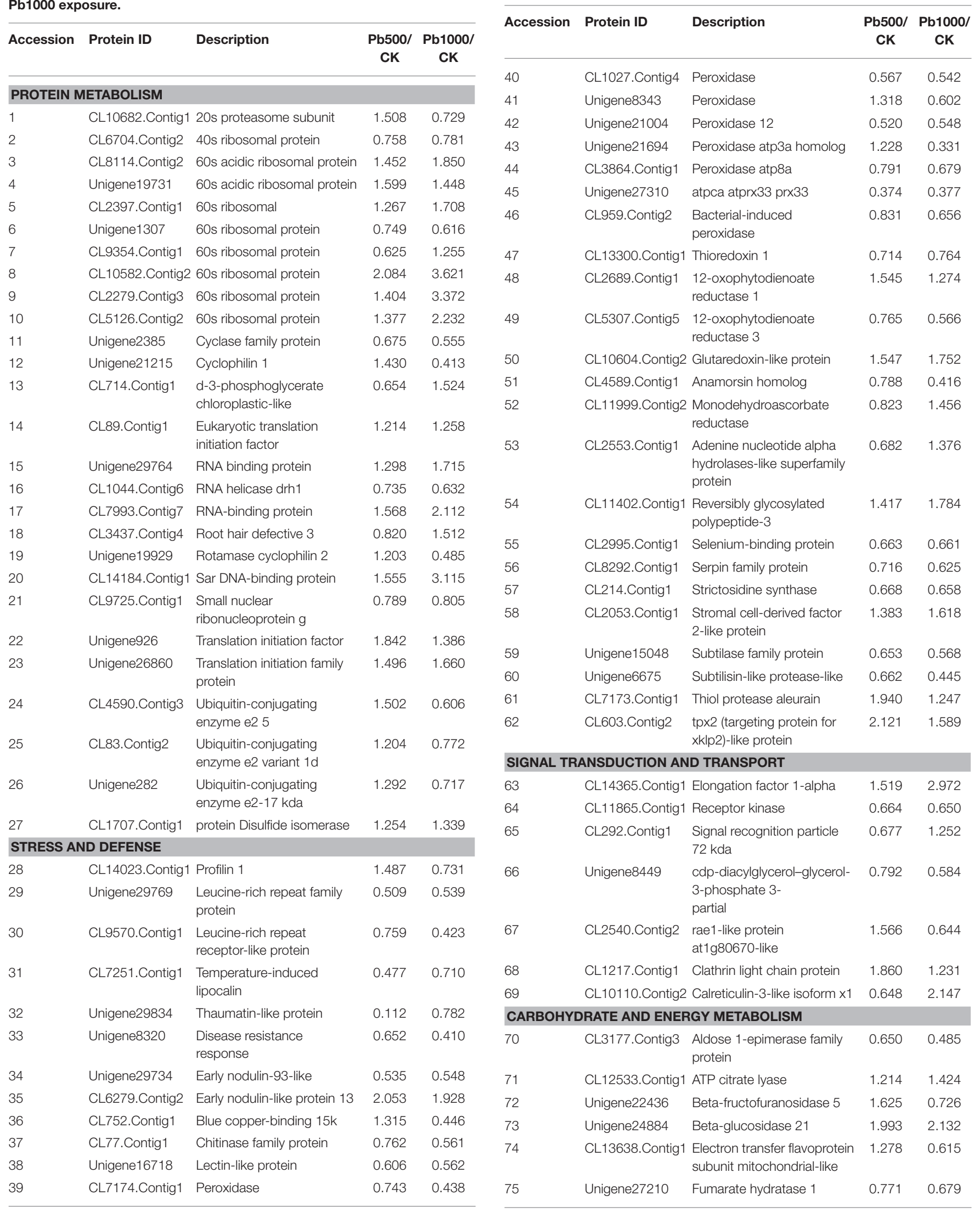

(Continued)
TABLE 1 | Continued

(Continued) 
TABLE 1 | Continued

\begin{tabular}{|c|c|c|c|c|}
\hline Accession & Protein ID & Description & $\begin{array}{l}\mathrm{Pb} 500 / \\
\mathrm{CK}\end{array}$ & $\begin{array}{l}\mathrm{Pb} 1000 / \\
\mathrm{CK}\end{array}$ \\
\hline 76 & Unigene20278 & $\begin{array}{l}\text { Fumarate hydratase } \\
\text { mitochondrial-like }\end{array}$ & 0.671 & 0.569 \\
\hline 77 & CL10499.Contig1 & $\begin{array}{l}\text { Glucan endo- } \\
\text {-beta-glucosidase } 4\end{array}$ & 1.252 & 0.441 \\
\hline 78 & Unigene25466 & $\begin{array}{l}\text { Glucan endo- } \\
\text {-beta-glucosidase 6-like }\end{array}$ & 1.268 & 0.659 \\
\hline 79 & CL4038.Contig1 & Isocitrate dehydrogenase & 0.803 & 0.743 \\
\hline 80 & Unigene23018 & Malate dehydrogenase & 0.778 & 0.735 \\
\hline 81 & CL8917.Contig1 & Myosin heavy chain kinase & 1.319 & 1.551 \\
\hline 82 & Unigene28617 & $\begin{array}{l}\mathrm{NADH} \text {-cytochrome b5 } \\
\text { reductase-like }\end{array}$ & 1.244 & 0.666 \\
\hline 83 & CL6783.Contig1 & $\begin{array}{l}\text { Pyruvate dehydrogenase } \\
\text { family protein }\end{array}$ & 0.724 & 0.819 \\
\hline 84 & CL6151.Contig2 & $\begin{array}{l}\text { Protein weak chloroplast } \\
\text { movement under blue light } \\
\text { 1-like }\end{array}$ & 1.657 & 2.037 \\
\hline 85 & CL5706.Contig6 & Transketolase & 1.426 & 5.285 \\
\hline 86 & CL859.Contig3 & Transaldolase-like protein & 0.808 & 1.288 \\
\hline 87 & CL5075.Contig1 & $\begin{array}{l}\text { Succinate-semialdehyde } \\
\text { mitochondrial-like isoform } \\
x 1\end{array}$ & 0.790 & 0.811 \\
\hline 88 & CL3677.Contig1 & $\begin{array}{l}\text { Aconitase c-terminal } \\
\text { domain-containing protein }\end{array}$ & 1.266 & 0.805 \\
\hline 89 & CL452.Contig1 & $\begin{array}{l}\text { Beta-xylosidase } \\
\text { alpha---arabinofuranosidase } \\
\text { 2-like }\end{array}$ & 0.524 & 0.526 \\
\hline 90 & CL452.Contig4 & $\begin{array}{l}\text { Beta-xylosidase } \\
\text { alpha-l-arabinofuranosidase } \\
\text { 2-like }\end{array}$ & 0.754 & 0.451 \\
\hline 91 & CL7567.Contig2 & $\begin{array}{l}\text { Dihydrolipoyl } \\
\text { dehydrogenase } 2\end{array}$ & 0.705 & 1.526 \\
\hline \multicolumn{5}{|c|}{ AMINO ACID AND LIPIDS METABOLISM } \\
\hline 92 & Unigene10784 & $\begin{array}{l}\text { Macrophage migration } \\
\text { inhibitory factor family } \\
\text { protein }\end{array}$ & 1.567 & 0.560 \\
\hline 93 & Unigene24925 & $\begin{array}{l}\text { gdsl esterase lipase } \\
\text { at1g54790-like }\end{array}$ & 2.379 & 0.683 \\
\hline 94 & Unigene8067 & $\begin{array}{l}\text { gdsl esterase lipase } \\
\text { at3g26430-like }\end{array}$ & 0.540 & 0.450 \\
\hline 95 & Unigene22679 & Glycine dehydrogenase & 0.787 & 0.768 \\
\hline 96 & Unigene25700 & $\begin{array}{l}\text { Glycosyl hydrolase family } 38 \\
\text { protein }\end{array}$ & 0.738 & 0.572 \\
\hline 97 & Unigene24148 & $\begin{array}{l}\text { Xylem cysteine proteinase } \\
1 \text {-like }\end{array}$ & 0.811 & 0.566 \\
\hline 98 & CL9552.Contig2 & Methyltransferase pmt24 & 1.336 & 1.571 \\
\hline 99 & Unigene2767 & $\begin{array}{l}\text { Myosin-related family } \\
\text { protein }\end{array}$ & 1.327 & 1.358 \\
\hline 100 & CL12773.Contig2 & $\begin{array}{l}\text { Nascent } \\
\text { polypeptide-associated } \\
\text { complex subunit alpha-like }\end{array}$ & 1.602 & 0.562 \\
\hline 101 & CL2541.Contig2 & $\begin{array}{l}\text { Serine } \\
\text { carboxypeptidase-like } 29\end{array}$ & 3.161 & 1.950 \\
\hline 102 & Unigene2790 & Aminomethyltransferase & 0.601 & 0.585 \\
\hline 103 & CL783.Contig2 & $\begin{array}{l}\text { Peptidyl-prolyl cis-trans } \\
\text { isomerase cyp20-3 }\end{array}$ & 0.566 & 0.629 \\
\hline
\end{tabular}

(Continued)
TABLE 1 | Continued

\begin{tabular}{|c|c|c|c|c|}
\hline Accession & Protein ID & Description & $\begin{array}{l}\mathrm{Pb} 500 / \\
\mathrm{CK}\end{array}$ & $\begin{array}{l}\mathrm{Pb} 1000 / \\
\mathrm{CK}\end{array}$ \\
\hline 104 & CL2368.Contig1 & $\begin{array}{l}\text { Proteasome subunit alpha } \\
\text { type-2-b }\end{array}$ & 1.292 & 0.574 \\
\hline 105 & Unigene289 & s9 Tyrosyl aminopeptidase & 1.606 & 2.699 \\
\hline 106 & CL6065.Contig3 & Aspartic proteinase & 0.511 & 0.612 \\
\hline 107 & CL9279.Contig1 & $\begin{array}{l}\text { Aspartyl protease family } \\
\text { protein }\end{array}$ & 0.503 & 0.179 \\
\hline 108 & CL5981.Contig1 & $\begin{array}{l}\text { Dimethylmenaquinone } \\
\text { methyltransferase family } \\
\text { protein }\end{array}$ & 1.301 & 0.408 \\
\hline 109 & CL330.Contig1 & Cupin family protein & 0.714 & 0.522 \\
\hline 110 & CL2774.Contig2 & Dehydrin erd14 & 1.678 & 1.539 \\
\hline \multicolumn{5}{|c|}{ CELL WALL AND CYTOSKELETON } \\
\hline 111 & CL2803.Contig4 & $\begin{array}{l}\text { Polygalacturonase inhibiting } \\
\text { protein }\end{array}$ & 0.383 & 0.491 \\
\hline 112 & CL12269.Contig1 & $\begin{array}{l}\text { Polygalacturonase-like } \\
\text { protein }\end{array}$ & 0.703 & 0.502 \\
\hline 113 & CL1120.Contig1 & $\begin{array}{l}\text { GTP-binding protein } \\
\text { sar1a-like }\end{array}$ & 1.252 & 1.646 \\
\hline 114 & CL741.Contig1 & Nuclear RNA binding & 1.847 & 1.539 \\
\hline 115 & CL14456.Contig1 & Neurofilament protein & 3.295 & 1.200 \\
\hline 116 & CL5177.Contig3 & Nuclear protein & 1.503 & 2.413 \\
\hline 117 & CL319.Contig1 & $\begin{array}{l}\text { Nucleic acid binding isoform } \\
\text { partial }\end{array}$ & 1.319 & 1.582 \\
\hline 118 & CL12221.Contig1 & Nucleolin family protein & 1.464 & 1.674 \\
\hline 119 & CL1396.Contig2 & Nucleolin like 1 & 1.550 & 1.814 \\
\hline 120 & CL4932.Contig1 & $\begin{array}{l}\text { Pectinacetylesterase family } \\
\text { protein }\end{array}$ & 1.306 & 0.638 \\
\hline 121 & Unigene6860 & Pectinesterase inhibitor & 0.630 & 0.563 \\
\hline 122 & CL2232.Contig2 & $\begin{array}{l}\text { Probable pectinesterase } \\
\text { pectinesterase inhibitor } \\
\text { 51-like }\end{array}$ & 0.823 & 0.627 \\
\hline 123 & CL1396.Contig2 & Nucleolin like 1 & 1.550 & 1.814 \\
\hline 124 & Unigene21122 & GPI-anchored protein & 0.695 & 0.420 \\
\hline 125 & CL2657.Contig1 & $\begin{array}{l}\text { Lipid-associated family } \\
\text { protein }\end{array}$ & 1.459 & 0.475 \\
\hline 126 & Unigene25152 & Vesicle associated protein & 1.618 & 1.678 \\
\hline 127 & Unigene22796 & $\begin{array}{l}\text { Organellar DNA-binding } \\
\text { protein } 1\end{array}$ & 0.756 & 0.544 \\
\hline 128 & CL8050.Contig1 & Partial & 1.295 & 0.681 \\
\hline 129 & Unigene2988 & $\begin{array}{l}\text { O-glycosyl hydrolases family } \\
17 \text { protein }\end{array}$ & 1.529 & 0.726 \\
\hline 130 & Unigene29720 & Endochitinase isolog & 0.619 & 0.651 \\
\hline 131 & Unigene29711 & $\begin{array}{l}\text { Adenine nucleotide alpha } \\
\text { hydrolases-like protein }\end{array}$ & 0.698 & 0.472 \\
\hline 132 & CL3373.Contig1 & Luminal binding protein & 1.210 & 1.573 \\
\hline \multicolumn{5}{|c|}{ UNCHARACTERIZED PROTEIN } \\
\hline 133 & CL12123.Contig1 & Unknown & 3.464 & 1.979 \\
\hline 134 & CL8554.Contig2 & Uncharacterized protein & 1.310 & 0.677 \\
\hline 135 & CL14500.Contig1 & Uncharacterized protein & 1.643 & 0.763 \\
\hline
\end{tabular}

during Pb500 and Pb1000 exposures. Among them, nine ribosomal protein species were up-accumulated under either one or both $\mathrm{Pb}$ treatments, while only one ribosomal component was both down-accumulated during the two Pb-stressed conditions. 
TABLE 2 | The dominant enriched GO terms for the 135 common DAPS under Pb500 and Pb1000 exposures.

\begin{tabular}{|c|c|c|c|c|c|}
\hline Go term & ID & Input number & Background number & $P$-Value & Corrected $\boldsymbol{P}$-Value \\
\hline Cell wall & GO:0005618 & 24 & 480 & 4.69E-12 & 2.89E-09 \\
\hline Apoplast & GO:0048046 & 18 & 267 & $2.21 \mathrm{E}-11$ & 9.07E-09 \\
\hline Response to metal ion & GO:0010038 & 17 & 425 & $1.25 \mathrm{E}-07$ & 2.77E-05 \\
\hline Vacuole & GO:0005773 & 21 & 650 & $1.42 \mathrm{E}-07$ & 2.77E-05 \\
\hline Peroxidase activity & GO:0004601 & 8 & 68 & 1.62E-07 & 2.77E-05 \\
\hline Plasmodesma & GO:0009506 & 20 & 645 & 5.16E-07 & 5.43E-05 \\
\hline Organelle lumen & GO:0043233 & 15 & 373 & 6.29E-07 & 5.54E-05 \\
\hline Membrane-enclosed lumen & GO:0031974 & 15 & 376 & 6.93E-07 & 5.69E-05 \\
\hline Antioxidant activity & GO:0016209 & 8 & 86 & 8.46E-07 & 6.51E-05 \\
\hline Response to inorganic substance & GO:0010035 & 25 & 1085 & 4.04E-06 & 0.000293 \\
\hline Cytosol & GO:0005829 & 27 & 1270 & 7.07E-06 & 0.000484 \\
\hline Nucleolus & GO:0005730 & 10 & 208 & 1.04E-05 & 0.000677 \\
\hline Extracellular region & GO:0005576 & 40 & 2386 & 1.48E-05 & 0.00091 \\
\hline Plant-type cell wall & GO:0009505 & 10 & 240 & 3.37E-05 & 0.001442 \\
\hline Positive regulation by symbiont of host innate immune response & GO:0052166 & 3 & 6 & 3.86E-05 & 0.001442 \\
\hline
\end{tabular}

TABLE 3 | The dominant pathways for the 135 common DAPS under Pb500 and Pb1000 exposure.

\begin{tabular}{|c|c|c|c|c|c|}
\hline Term & Database & Input number & Background number & P-Value & Corrected $\boldsymbol{P}$-Value \\
\hline The citric acid (TCA) cycle and respiratory electron transport & Reactome & 5 & 26 & 2.30E-05 & 0.001287 \\
\hline Citrate cycle (TCA cycle) & KEGG PATHWAY & 7 & 60 & 0.000112 & 0.002884 \\
\hline Phenylalanine metabolism & KEGG PATHWAY & 9 & 106 & 0.000117 & 0.002933 \\
\hline Pyruvate metabolism and Citric Acid (TCA) cycle & Reactome & 4 & 22 & 0.000192 & 0.004371 \\
\hline Phenylpropanoid biosynthesis & KEGG PATHWAY & 10 & 145 & 0.000243 & 0.005078 \\
\hline Citric acid cycle (TCA cycle) & Reactome & 3 & 10 & 0.000369 & 0.007341 \\
\hline Carbon metabolism & KEGG PATHWAY & 11 & 217 & 0.001399 & 0.022388 \\
\hline
\end{tabular}

The abundance levels for three translation initiation family proteins (CL89.Contig1, Unigene926 and Unigene26860), two RNA binding proteins (Unigene29764 and CL7993.Contig7) and one protein disulfide isomerase (CL1707.Contig1) were observed to be increased under these two $\mathrm{Pb}$ exposures. However, one RNA helicase (CL1044.Contig6) was identified to be decreased responding to these two $\mathrm{Pb}$ treatments. All three identified ubiquitin-conjugating enzymes (CL4590.Contig3, CL83.Contig2, and Unigene282) and one cyclase family protein (Unigene2385) were coordinately increased at the $\mathrm{Pb} 500$ exposure while decreased at the Pb1000 (Table 1).

It is known that the antioxidant enzymes could ensure cellular protection from the reactive oxygen species (ROS) mediated damage in plant responding to various environmental stresses. In the present study, the abundance changes for many antioxidant enzymes were detected including two 12-oxophytodienoate reductases (CL2689.Contig1 and CL5307.Contig5), one glutaredoxin-like protein (CL10604.Contig2), one thioredoxin (CL13300.Contig1) and eight peroxidases (CL7174.Contig1, CL1027.Contig4, Unigene8343, Unigene21004, Unigene21694, CL3864.Contig1, Unigene27310, and CL959.Contig2). Among these identified DAPS for antioxidant enzymes, most of them were decreased in radish under the exposure of $\mathrm{Pb}$ except one OPR (CL2689.Contig1) and one GR (CL10604.Contig2) up-accumulated at both $\mathrm{Pb}$ treatment, and two peroxidases (Unigene 8343 and Unigene 21694) up-accumulated under the Pb500 exposure (Table 1).

Additionally, a lot of protein species involved in carbohydrate and energy metabolism-related pathways (i.e., "TCA cycle and respiratory electron transport," "pyruvate metabolism," and "carbon metabolism") were shown to exhibit abundance changes under $\mathrm{Pb}$ exposure. The activities of most metabolic enzymes involved in these pathways were both repressed in the two concentrations of $\mathrm{Pb}$ treatments, including malate dehydrogenase (Unigene23018), endochitinase (Unigene29720), lectin-like protein (Unigene16718), polygalacturonase-like protein (CL12269.Contig1), aldose 1-epimerase family protein (CL3177.Contig3), beta-xylosidase alpha-l-arabinofuranosidase 2-like (CL452.Contig1), chitinase family protein (CL77.Contig1), and glycosyl hydrolase family 38 protein (Unigene25700). However, other important enzymes showed differentially accumulated during the two $\mathrm{Pb}$ stresses. For example, the expression levels of the acid invertase beta-fructofuranosidase 5 (Unigene22436), a beta-glucosidase glucan endo-betaglucosidase 4 (CL10499.Contig1), and a hydrolases o-glycosyl hydrolases family 17 protein (Unigene2988) were increased in $\mathrm{Pb} 500$ exposure, but decreased in $\mathrm{Pb} 1000$ stress (Table 1). 


\section{Associated Analysis of Pb-Responsive Genes through Proteomic in Combination with Other-Omic Techniques of Radish in Response to $\mathrm{Pb}$ Stress}

In order to investigate whether the protein level is correlated with the corresponding mRNA level alterations, the proteomic data was compared with our previous transcriptome data between CK vs. Pb1000 (Wang et al., 2013). Proteins were considered to be correlated when quantified proteins have expression information at the transcriptome level, and a total of 1667 protein species $(77.9 \%$ of all the quantified proteins) were detected from the transcriptome data (Table S4). Out of the 510 protein species found to be significantly accumulated under Pb1000 stress of radish, there were only 57 DAPS could be matched with their cognate differentially expressed genes (DEGs) including 25 regulated in the same trends and 32 found in the opposite direction (Table S5). In addition, there were 343 DAPS exhibiting no change in mRNA expression and 144 DEGs without altered expression at protein level (Table S5). These results indicated that the accumulation of transcripts and proteins occur independently. A generally low congruency of proteomic and transcriptional profiles has been reported in other previous studies (Lan et al., 2011; Zhuang et al., 2013).

Increasing evidences have revealed that miRNA-mediated gene regulation play significant roles in plant response to HM stress, and $19 \mathrm{~Pb}$-responsive miRNAs and their corresponding target mRNAs were identified in radish by siRNA sequencing and degradome analysis (Wang et al., 2015a). By exploring an integrated expression analysis of miRNA, mRNAs and proteins, a total of seven miRNA-mRNA pairs and matching proteins were identified in radish response to $\mathrm{Pb}$ stress, which mainly included two miRNA families, miR156 and miR396. As shown in Table 4, almost all Pb-responsive miRNAs and their corresponding mRNA targets had an anti-relationship at protein level. For example, two members of miR156 family (miR156b and miR156c) were found to be up-regulated in response to $\mathrm{Pb}$ exposure, while the expressions of their targets (glutaredoxin, aldose 1-epimerase and malate dehydrogenase) were all repressed at protein levels. Two corresponding protein species for the down-regulated miR396b were identified during $\mathrm{Pb}$ stress of radish, one (Translation initiation factor eIF-3) was up-accumulated, while the other one (thioredoxin-like 1-2, chloroplastic) was shown to be down-accumulated.

For deeply dissecting the molecular mechanism underlying $\mathrm{Pb}$ tolerance and homeostasis in radish, we further performed an association analysis of the seven Pb-responsive miRNA-mRNAproteins pairs with the differentially regulated metabolites identified in radish roots during $\mathrm{Pb}$ stress (Wang et al., 2015b). Based on the intersected pathway analysis, three $\mathrm{Pb}$-responsive metabolites including glucose, citrate and malate were found to be linked with the miRNA-mRNA-proteins, which may play a significant role in radish response to $\mathrm{Pb}$ stress (Table S6).

\section{DISCUSSION}

Proteomic technique provides a powerful tool for the analysis of molecular mechanism of plant response against stresses, and a path for linking gene expression to cell metabolism in the rapidly processing post-genome era (Ahsan et al., 2009; Liang et al., 2013). Increasing evidences have revealed that proteomic studies are playing important roles in the post-genomic era for characterizing the molecular mechanisms underlying plant responses to HM stresses (Fukao et al., 2011; Wang et al., 2013, 2014; Sebastiani et al., 2014). Radish is an important root vegetables worldwide, which has been considered as one of the most significant root species for dissecting the molecular regulatory network of HM stress in Brassicaceae crops (Xu et al., 2012; Wang et al., 2014, 2015a; Liu et al., 2015; Xie et al., 2015). In the current study, comparative proteomics analysis by iTRAQ together with other-omics techniques reveals complex regulatory network and provides insights into the response of radish root under $\mathrm{Pb}$ exposure. To the best of our knowledge, this is the first study to systematically investigate and characterize the protein abundance changes under $\mathrm{Pb}$ stress exposure in radish root with iTRAQ-based proteomics analysis.

\section{Proteins of Signal Sensing Mechanisms Involved in Radish Root Response to $\mathrm{Pb}$ Stress}

It was known that the cell wall could activate a variety of specific stress-responsive signaling proteins when perceive outer stress conditions (Jamet et al., 2006; Day et al., 2013). In previous

TABLE 4 | Association analysis of miRNAs, genes, and proteins responsive to $\mathrm{Pb}$ stress in radish root.

\begin{tabular}{|c|c|c|c|c|c|}
\hline miRNA & $\operatorname{LogFC}^{a}$ & Target mRNA annotation & Protein ID & $\operatorname{LogFC}^{b}$ & $\operatorname{LogFC}^{c}$ \\
\hline miR156b & 1.1813 & Glutaredoxin & CL10604.Contig2 & -0.12 & -0.247 \\
\hline $\operatorname{miR} 156 b$ & 1.1813 & Aldose 1-epimerase & CL3177.Contig3 & -0.19 & -0.314 \\
\hline miR156c & 1.0788 & Malate dehydrogenase & Unigene23018 & -0.11 & -0.134 \\
\hline miR396b & -0.5131 & Thioredoxin & CL13300.Contig1 & -0.15 & -0.117 \\
\hline miR396b & -0.5131 & Translation initiation factor & CL89.Contig1 & 0.084 & 0.0997 \\
\hline miR396b & -0.5131 & Translation initiation factor & Unigene926 & 0.265 & 0.1418 \\
\hline miR396b & -0.5131 & Translation initiation factor & Unigene26860 & 0.175 & 0.2201 \\
\hline
\end{tabular}

a miRNA fold change under Pb500.

${ }^{b}$ Protein fold change under Pb500.

${ }^{c}$ Protein fold change under Pb1000. 
studies, a set of cell wall-related protein species exhibited dynamic changes in response to $\mathrm{HM}$ stress, such as $\mathrm{Al}$ exposure in rice and cadmium $(\mathrm{Cd})$ exposure in flax (Hradilová et al., 2010; Wang et al., 2013, 2014). Currently, "cell wall" (GO: 0005618) was identified as the most enriched GO term for all the 135 common DAPS involved in the Pb500 and Pb1000 stress, which indicated that cell wall may play a vital role in response to $\mathrm{Pb}$ stress of radish. Two critical inhibiting protein species including a pectinesterase inhibitor (CL2232.Contig2) and a polygalacturonase inhibitor (CL2803.Contig4) were both downaccumulated during the stresses of $\mathrm{Pb} 500$ and $\mathrm{Pb} 1000$ in radish. The two protein species were verified to modulate the activities of pectinesterases (PE) and polygalacturonases (PG), which could help the plant to fine-tune cell wall remodeling processes when exposure stress conditions (Pelloux et al., 2007; Ferrari et al., 2012). The down-accumulation of the inhibiting proteins maybe result in up- accumulation of $\mathrm{PE}$ and $\mathrm{PG}$, indicating their positive role to the cell wall remodeling when encounter the $\mathrm{Pb}$ stress of radish.

Calreticulin (CRT) is an abundant endo reticulum $\mathrm{Ca}^{2+}$ binding protein, and plays a critical role in $\mathrm{Ca}^{2+}$ homeostasis and signaling sensing network (Corbett and Michalak, 2000; Jia et al., 2009). In this study, one CRT (calreticulin-3-like isoform $\mathrm{x} 1$, CL10110.Contig2) was found to be induced by the Pb1000, indicating that the altered expression of CRT maybe function as signaling molecular in modulating the radish plant to adapt to the $\mathrm{Pb}$-stressed environments. Additionally, jasmonate (JA) is known to be a vital signaling molecule which can be activated in response to a wide range of environmental cues including heavy metals (Maksymiec et al., 2005; Schommer et al., 2008; Liu et al., 2009). In this study, the expression level of 12oxo-phytodienoic acid reductase (OPR), a key enzyme involved in the JA biosynthesis pathway, showed differential abundance levels during the Pb stress. The OPR-1 (CL2689.Contig1) was up-accumulated in radish roots when subjected to the either $\mathrm{Pb} 500$ or Pb1000 stress, and a similar result was found at the proteome level in rice root exposure to As stress (Ahsan et al., 2008; Srivastava et al., 2009). However, the expression level of OPR-3 (CL5307.Contig5) was decreased in radish roots under the conditions of $\mathrm{Pb} 500$ and $\mathrm{Pb} 1000$ exposure, indicating that different members of the OPR were differentially accumulated under the $\mathrm{Pb}$ exposure in plant.

\section{Proteins of Carbohydrate and Energy Metabolism Involved in Radish Root Response to $\mathrm{Pb}$ Stress}

The accumulation of HM in plants can severely affect the photosynthetic pathway and thus lead to symptoms of toxicity, such as chlorosis and growth reduction (Pourrut et al., 2013). To maintain the normal growth and development, or at least to protect the cells against excess damages, plants need to activate the carbohydrate and energy metabolism related metabolic pathways (Sarry et al., 2006; Thapa et al., 2012). In this study, "citric acid (TCA) cycle and respiratory electron transport" was identified as the most enriched pathway, and a lot of critical metabolic enzymes were shown to exhibit abundance changes under $\mathrm{Pb}$ exposure. Beta-glucosidase catalyzes the hydrolysis of the glycosidic and release of glucose, which then entry into the glycolysis process. ATP-citrate lyase (ACL, EC4.1.3.8) is a key enzyme involved in TCA cycle, which catalyzes the cleavage of citrate to yield acetyl CoA, oxaloacetate, ADP, and orthophosphate, and the ACL gene was found to be induced under various stresses including low temperature and drought stimulus ( $\mathrm{Hu}$ et al., 2015). Pyruvate dehydrogenase could exert a key role in linking the glycolysis to the TCA cycle by catalyzing the formation of an acetyl-CoA from pyruvate (Vuoristo et al., 2015), which is a vital rate-limiting step reaction that determines the rate and efficiency of TCA cycle. In this study, a beta-glucosidase 21 (Unigene24884), ATP citrate lyase (ACL12533.Contig1), and a pyruvate dehydrogenase (CL6783.Contig1) were observed to be both up-accumulated during the $\mathrm{Pb} 500$ and $\mathrm{Pb} 1000$ stress of radish root, which revealed their positive potential role in producing more reducing power to compensate high-energy demand response to $\mathrm{Pb}$ stress. However, the abundances of the some other metabolic enzymes including Isocitrate dehydrogenase (CL4038.Contig1), fumarate hydratase (Unigene27210, Unigene20278), and malate dehydrogenase (Unigene23018) were decreased under the $\mathrm{Pb}$-stresses, indicating their activity suffered repression. These decreased proteins may influence the biosynthesis and accumulation of organic acids such as citrate and malate, which play critical roles in HM tolerance (Ma et al., 2001; Wang et al., 2015b).

\section{Proteins of Antioxidative Defense and Detoxification Involved in Radish Root Response to $\mathbf{P b}$ Stress}

The presence of excess metal ions causes ROS in plants, which can irreversibly damage the cells and attack macromolecules (Ahsan et al., 2009). However, the ROS can be scavenged by plant antioxidant defense system consisting of antioxidant compounds and enzymes (Apel and Hirt, 2004; Suzuki et al., 2012). The ascorbate (ASA)-glutathione (GSH) cycle is one of the main antioxidant systems in plants to keep ROS under control being, which involves a lot of critical antioxidant enzymes in a series of cyclic reactions to detoxify $\mathrm{H}_{2} \mathrm{O}_{2}$ (de Sousa et al., 2016; Noshi et al., 2016). One of the antioxidant enzymes is peroxidase (POD), which can detoxify $\mathrm{H}_{2} \mathrm{O}_{2}$ by oxidizing ascorbate. It was reported that different members of the peroxidase gene family were differentially accumulated under various HM exposures in Arabidopsis (Kumari et al., 2008), wheat (Houde and Diallo, 2008) and aspen (Grisel et al., 2010). In this study, the term of "peroxidase activity" (GO: 0004601) was identified as one of the most over-represented GO terms and six POD protein species were identified as DAPS, indicating the POD may be the critical ROS-scavenging protein in radish root response to $\mathrm{Pb}$ stress. The abundance of another antioxidant enzyme involved in ASA-GSH cycle, monodehydroascorbate reductase (MDHAR, CL11999.Contig2) was also found to be altered in response to $\mathrm{Pb}$ stress. The main function of MDHAR is responsible for ascorbate regeneration in plant tissues, and the expression level changed during the HM stress in B. juncea root 
(Alvarez et al., 2009) and A. Halleri shoot (Farinati et al., 2009). Additionally, many thiol-containing antioxidants, peroxiredoxin (PRX33, unigene27310), glutaredoxin-like protein (GRX, CL10604.Contig2) and thioredoxin (TRX, CL13300.Contig1), were also found to be altered during the $\mathrm{Pb}$-stressed condition of radish root. The Prx is a thiol peroxidase with multiple functions, which was found to be induced under various HM stresses such as Cd (Hossain et al., 2013) and As (Pandey et al., 2012). Glutaredoxin (GRX) and thioredoxin (TRX) could be oxidized by peroxides and regenerate peroxiredoxins, which were verified to play direct roles in the antioxidative system by regenerating peroxiredoxins oxidized by peroxides (Hossain and Komatsu, 2013).

\section{The Genetic Regulatory Network of Radish Root Response to Pb Stress}

The mechanism of HM response is a complex process that a variety of genes and response components involved in plants (Fukao et al., 2011; Sebastiani et al., 2014). In the present study, to reveal the molecular mechanism underlying $\mathrm{Pb}$ stress response in radish root, a putative schematic network was put forward based on proteomic information of this study in conjunction with integrated analysis of miRNA, transcriptomic and metabolomic data (Figure 4). Once $\mathrm{Pb}$ enters the cell of radish plants, the cell wall firstly perceive stress signals and then activate specific stress-responsive signaling molecules including fine-tuning cell wall remodeling processes through special proteins like PE and PG, triggering the HM stress responsive hormones levels such as JA as well as modulating calcium-signaling molecules. With the aid of signaling molecules, the stress signals were transmitted and ultimately give rise to the alterations in gene expression and protein levels. A direct consequence of HM stress was the disturbance in the balance of protein synthesis and degradation, which is essential to both cellular homeostasis and dynamics because almost all biological processes need the involvement of enzymes. Under Pb stress, many key enzymes (i.e., ATP citrate lyase, Isocitrate dehydrogenase, fumarate hydratase and malate dehydrogenase) involved in the glycolysis and TCA cycle were severely affected, which ultimately cause alteration of some metabolites including glucose, citrate and malate. The glucose could act as the osmoprotectants protecting the cell constituents, and the organic acids (such as citrate and malate) played critical roles in chelating toxic HM ions. Meanwhile, a series of other defense responses were triggered to cope with $\mathrm{Pb}$-induced injuries. For example, the ASA-GSH cycle was the main antioxidant systems for scavenging the accumulated ROS to alleviate oxidative damages. Moreover, several $\mathrm{Pb}$ defense protein species (glutaredoxin, aldose 1-epimerase malate dehydrogenase and thioredoxin) encoding genes targeted by miRNA156 and miR396, which were found to play critical

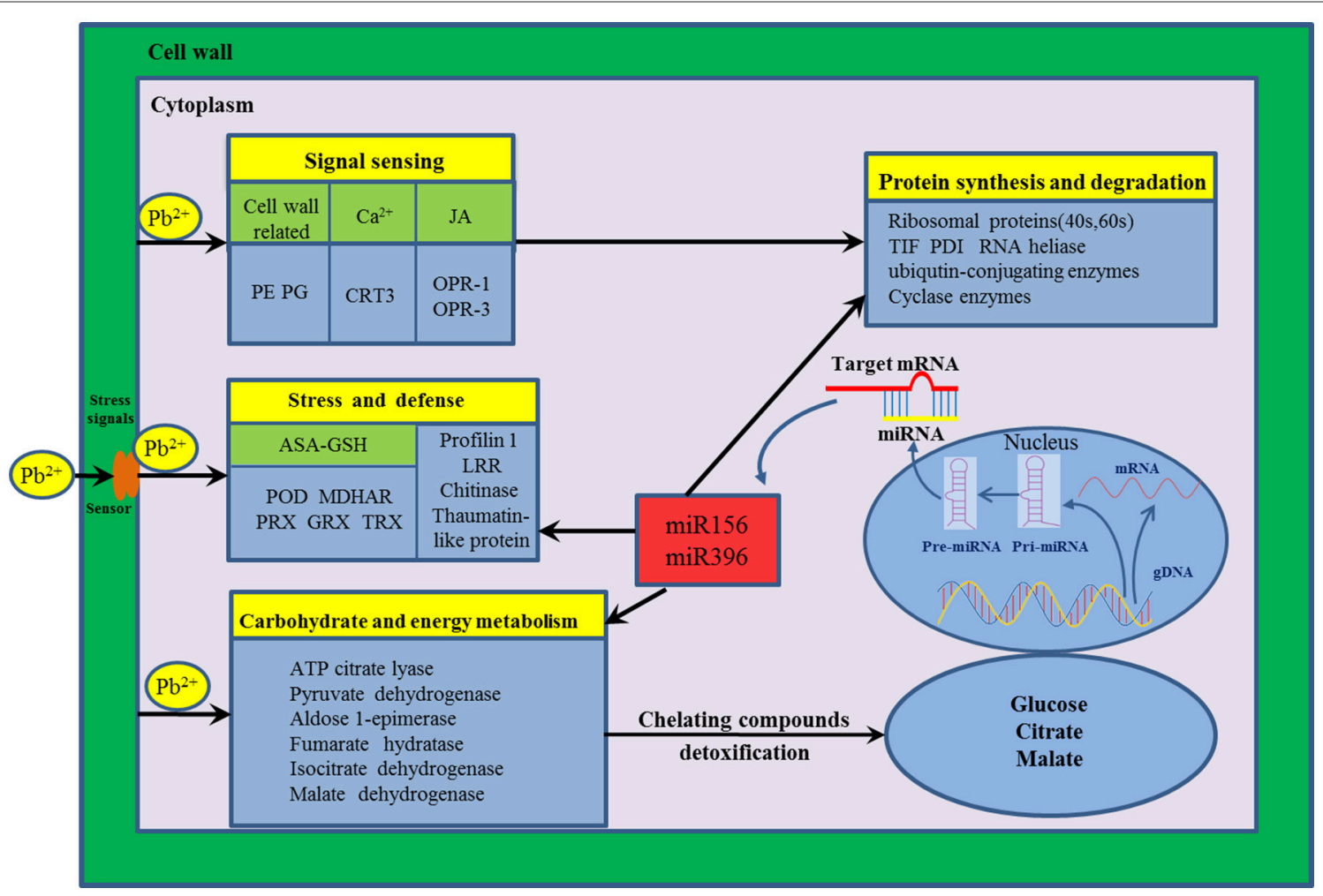

FIGURE 4 | The putative genetic regulatory network of Pb stress response in radish root. 12-oxo-phytodienoic acid reductase-1 (OPR-1), 12-oxo-phytodienoic acid reductase-3 (OPR-3), Ascorbate (ASA)-Glutathione (GSH), Calreticulin-3-like isoform X1 (CRT3), Glutaredoxin (GRX), thioredoxin (TRX), Leucine-rich repeat family protein (LRR), Monodehydroascorbate reductase (MDHAR), Pectinesterases (PE), Peroxidase (POD), Peroxiredoxin (PRX), Polygalacturonases (PG), Protein disulfide isomerase (PDI), Translation initiation factor (TIF). 
roles in defense system when radish root responds to $\mathrm{Pb}$ stress.

In summary, this is the first report on systematically investigating and characterizing the protein abundance changes under $\mathrm{Pb}$ stress in radish root with the iTRAQ technique. A total of 3898 protein species were successfully detected and 2141 were quantified. A subset of 721 protein species were differentially accumulated upon at least one $\mathrm{Pb}$ exposure, and 135 ones showed common abundance alterations under two $\mathrm{Pb}$-stressed conditions. GO and pathway enrichment analysis revealed that these 135 common DAPS were strongly enriched in the categories of cell structure, carbohydrate and energy metabolism-related pathways and antioxidative defense. Furthermore, the integrative analysis of transcriptomic, miRNA, degradome and metabolomic with proteomic data provided a strengthened understanding of radish root response to $\mathrm{Pb}$ stress, and a schematic genetic regulatory network was put forward. The genes associated with signal sensing, protein synthesis and degradation, carbohydrate and energy metabolism and ASA-GSH cycle for ROS scavenging as well as several key miRNAs and metabolites were crucially responsible for radish in response to $\mathrm{Pb}$ stress. Overall, the results of this study would be beneficial for further dissecting molecular mechanisms underlying other plant responses to HM stresses, and facilitate genetically effective management of HM contamination in root vegetable crops.

\section{AUTHOR CONTRIBUTIONS}

YW designed the experiments and drafted the manuscript. LX, MT and WZ participated in the design of the study

\section{REFERENCES}

Ahsan, N., Lee, D. G., Alam, I., Kim, P. J., Lee, J. J., Ahn, Y. O., et al. (2008). Comparative proteomic study of arsenic-induced differentially expressed proteins in rice roots reveals glutathione plays a central role during As stress. Proteomics 8, 3561-3576. doi: 10.1002/pmic.200701189

Ahsan, N., Renaut, J., and Komatsu, S. (2009). Recent developments in the application of proteomics to the analysis of plant responses to heavy metals. Proteomics 9, 2602-2621. doi: 10.1002/pmic.200800935

Alvarez, S., Berla, B. M., Sheffield, J., Cahoon, R. E., Jez, J. M., and Hicks, L. M. (2009). Comprehensive analysis of the Brassica juncea root proteome in response to cadmium exposure by complementary proteomic approaches. Proteomics 9, 2419-2431. doi: 10.1002/pmic.200800478

Apel, K., and Hirt, H. (2004). Reactive oxygen species: metabolism, oxidative stress, and signal transduction. Annu. Rev. Plant Biol. 55, 373-399. doi: 10.1146/annurev.arplant.55.031903.141701

Bindschedler, L. V., and Cramer, R. (2011). Quantitative plant proteomics. Proteomics 11, 756-775. doi: 10.1002/pmic.201000426

Conesa, A., and Götz, S. (2008). Blast2GO: a comprehensive suite for functional analysis in plant genomics. Int. J. Plant Genomics 2008:619832. doi: 10.1155/2008/619832.

Conesa, A., Götz, S., García-Gömez, J. M., Terol, J., Talón, M., and Robles, M. (2005). Blast2GO: a universal tool for annotation, visualization and analysis in functional genomics research. Bioinformatics 21, 3674-3676. doi: 10.1093/bioinformatics/bti610

Corbett, E. F., Michalak, M. (2000). Calcium, a signaling molecule in the endoplasmic reticulum? Trends Biochem. Sci. 25, 307-311. doi: 10.1016/S0968-0004(00)01588-7 and performed the data analysis. MT, RW and HJ planted radish seedlings and collected samples. LX and WC reviewed and edited the manuscript. LL conceived of the study, and participated in its design and coordination and helped to draft the manuscript. All authors read and approved the final manuscript.

\section{ACKNOWLEDGMENTS}

This work was in part supported by grants from the NSFC (31601766, 31501759), NSF of Jiangsu Province (BK20140706), China Postdoctoral Science Foundation (2016T90472, 2015M570458, 2016M590466) and the National Key Technology Research and Development Program of China (2016YFD0100204-25).

\section{SUPPLEMENTARY MATERIAL}

\section{Table S1 | The DAPS involved in radish root response under $\mathrm{Pb500}$ exposure.}

Table S2 | The DAPS in radish root response under Pb1000 exposure.

Table S3 | KEGG pathways of the DAPS involved in radish root response under $\mathrm{Pb} 500$ and $\mathrm{Pb} 1000$ exposure.

Table S4 | The quantitative proteins detected in the transcriptomic analysis of radish under $\mathrm{Pb}$ exposure.

Table S5 | The correlated DAPS and DEGs involved in Pb stress response of radish.

Table S6 | Association analysis of miRNAs, genes, metabolites and proteins responsive to $\mathrm{Pb}$ stress in radish root.

Day, A., Fénart, S., Neutelings, G., Hawkins, S., Rolando, C., and Tokarski, C. (2013). Identification of cell wall proteins in the flax (Linum usitatissimum) stem. Proteomics 13, 812-825. doi: 10.1002/pmic.201200257

de Sousa, A., AbdElgawad, H., Han, A., Teixeira, J., Matos, M., and Fidalgo, F. (2016). Oxidative metabolism of rye (Secale cereale L.) after short term exposure to aluminum: uncovering the glutathione-ascorbate redox network. Front. Plant Sci. 7:685. doi: 10.3389/fpls.2016.00685

Farinati, S., DalCorso, G., Bona, E., Corbella, M., Lampis, S., Cecconi, D., et al. (2009). Proteomic analysis of Arabidopsis halleri shoots in response to the heavy metals cadmium and zinc and rhizosphere microorganisms. Proteomics 9, 4837-4850. doi: 10.1002/pmic.200900036

Ferrari, S., Sella, L., Janni, M., De Lorenzo, G., Favaron, F., and D’Ovidio, R. (2012). Transgenic expression of polygalacturonase-inhibiting proteins in Arabidopsis and wheat increases resistance to the flower pathogen Fusarium graminearum. Plant Biol. 14, 31-38. doi: 10.1111/j.1438-8677.2011.00449.x

Fu, Y., Zhang, H., Mandal, S. N., Wang, C., Chen, C., and Ji, W. (2016). Quantitative proteomics reveals the central changes of wheat in response to powdery mildew. J. Proteomics 130, 108-119. doi: 10.1016/j.jprot.2015.09.006

Fukao, Y., Ferjani, A., Tomioka, R., Nagasaki, N., Kurata, R., Nishimori, Y., et al. (2011). iTRAQ analysis reveals mechanisms of growth defects due to excess zinc in Arabidopsis. Plant Physiol. 155, 1893-1907. doi: 10.1104/pp.110.169730

Glibert, P., Van Steendam, K., Dhaenens, M., and Deforce, D. (2014). iTRAQ as a method for optimization: enhancing peptide recovery after gel fractionation. Proteomics 14, 680-684. doi: 10.1002/pmic.201300444

Grisel, N., Zoller, S., Künzli-Gontarczyk, M., Lampart, T., Münsterkötter, M., Brunner, I., et al. (2010). Transcriptome responses to aluminum stress in roots of aspen (Populus tremula). BMC Plant Biol. 10:185. doi: $10.1186 / 1471-2229-10-185$ 
Gupta, D. K., Huang, H. G., and Corpas, F. J. (2013). Lead tolerance in plants: strategies for phytoremediation. Environ. Sci. Pollut. Res. 20, 2150-2161. doi: 10.1007/s11356-013-1485-4

Gupta, D., Nicoloso, F., Schetinger, M., Rossato, L., Huang, H., Srivastava, S., et al. (2011). Lead induced responses of Pfaffia glomerata, an economically important Brazilian medicinal plant, under in vitro culture conditions. Bull. Environ. Contam. Toxicol. 86, 272-277. doi: 10.1007/s00128-011-0226-y

Hinkson, I. V., and Elias, J. E. (2011). The dynamic state of protein turnover: it's about time. Trends Cell Biol. 21, 293-303. doi: 10.1016/j.tcb.2011. 02.002

Hossain, Z., Khatoon, A., and Komatsu, S. (2013). Soybean Proteomics for Unraveling Abiotic Stress Response Mechanism. J. Proteome Res. 12, 4670-4684. doi: 10.1021/pr400604b

Hossain, Z., and Komatsu, S. (2013). Contribution of proteomic studies towards understanding plant heavy metal stress response. Front. Plant Sci. 3:310. doi: $10.3389 /$ fpls.2012.00310

Houde, M., and Diallo, A. O. (2008). Identification of genes and pathways associated with aluminum stress and tolerance using transcriptome profiling of wheat near-isogenic lines. BMC Genomics 9:400. doi: 10.1186/1471-2164-9-400

Hradilová, J., Řehulka, P., Řehulká, H., Vrbová, M., Griga, M., and Brzobohatý, B. (2010). Comparative analysis of proteomic changes in contrasting flax cultivars upon cadmium exposure. Electrophoresis 31, 421-431. doi: 10.1002/elps.200900477

Hu, X. M., Shi, C. Y., Liu, X., Jin, L. F., Liu, Y. Z., and Peng, S. A. (2015). Genomewide identification of citrus ATP-citrate lyase genes and their transcript analysis in fruits reveals their possible role in citrate utilization. Mol. Genet. Genomics 290, 29-38. doi: 10.1007/s00438-014-0897-2

Jamet, E., Canut, H., Boudart, G., and Pont-Lezica, R. F. (2006). Cell wall proteins: a new insight through proteomics. Trends Plant Sci. 11, 33-39. doi: 10.1016/j.tplants.2005.11.006

Jia, X. Y., He, L. H., Jing, R. L., and Li, R. Z. (2009). Calreticulin: conserved protein and diverse functions in plants. Physiol. Plant. 136, 127-138. doi: 10.1111/j.1399-3054.2009.01223.x

Kambiranda, D., Katam, R., Basha, S. M., and Siebert, S. (2013). iTRAQ-based quantitative proteomics of developing and ripening muscadine grape berry. J. Proteome Res. 13, 555-569. doi: 10.1021/pr400731p

Kumari, M., Taylor, G. J., and Deyholos, M. K. (2008). Transcriptomic responses to aluminum stress in roots of Arabidopsis thaliana. Mol. Genet. Genomics 279, 339-357. doi: 10.1007/s00438-007-0316-Z

Lan, P., Li, W., Wen, T. N., Shiau, J. Y., Wu, Y. C., Lin, W., et al. (2011). iTRAQ protein profile analysis of Arabidopsis roots reveals new aspects critical for iron homeostasis. Plant Physiol. 155, 821-834. doi: 10.1104/pp.110.169508

Li, W., Zhao, F. A., Fang, W. P., Xie, D. Y., Hou, J. N., Yang, X. J., et al. (2015). Identification of early salt stress responsive proteins in seedling roots of upland cotton (Gossypium hirsutum L.) employing iTRAQ-based proteomic technique. Front. Plant Sci. 6:732. doi: 10.3389/fpls.2015.00732

Liang, C., Tian, J., and Liao, H. (2013). Proteomics dissection of plant responses to mineral nutrient deficiency. Proteomics 13, 624-636. doi: 10.1002/pmic.201200263

Liu, L., Huang, H., Gong, Y., Chen, C., and Wang, L. (2009). Cytological and ultra-structural study on microsporogenesis of cytoplasmic male sterility in Raphanus sativus. Biologia 64, 716-722. doi: 10.2478/s11756-009-0063-1

Liu, W., Xu, L., Wang, Y., Shen, H., Zhu, X. W., Zhang, K. Y., et al. (2015). Transcriptome-wide analysis of chromium-stress responsive microRNAs to explore miRNA-mediated regulatory networks in radish (Raphanus sativus L.). Sci. Rep. 5:14024. doi: 10.1038/srep14024

Ma, J. F., Ryan, P. R., and Delhaize, E. (2001). Aluminium tolerance in plants and the complexing role of organic acids. Trends Plant Sci. 6, 273-278. doi: 10.1016/S1360-1385(01)01961-6

Maksymiec, W., Wianowska, D., Dawidowicz, A., Radkiewicz, S., Mardarowicz, M., and Krupa, Z. (2005). The level of jasmonic acid in Arabidopsis thaliana and Phaseolus coccineus plants under heavy metal stress. J. Plant Physiol. 162, 1338-1346. doi: 10.1016/j.jplph.2005.01.013

Martínez-Esteso, M. J., Vilella-Antón, M. T., Pedreño, M. Á., Valero, M. L., and Bru-Martínez, R. (2013). iTRAQ-based protein profiling provides insights into the central metabolism changes driving grape berry development and ripening. BMC Plant Biol. 13:167. doi: 10.1186/1471-2229-13-167
Noshi, M., Hatanaka, R., Tanabe, N., Terai, Y., Maruta, T., and Shigeoka, S. (2016). Redox regulation of ascorbate and glutathione by a chloroplastic dehydroascorbate reductase is required for high-light stress tolerance in Arabidopsis. Biosci. Biotechnol. Biochem. 80, 870-877. doi: 10.1080/09168451.2015.1135042

Pandey, S., Rai, R., and Rai, L. C. (2012). Proteomics combines morphological, physiological and biochemical attributes to unravel the survival strategy of Anabaena sp. PCC7120 under arsenic stress. J. Proteomics 75, 921-937. doi: 10.1016/j.jprot.2011.10.011

Pelloux, J., Rusterucci, C., and Mellerowicz, E. J. (2007). New insights into pectin methylesterase structure and function. Trends Plant Sci. 12, 267-277. doi: 10.1016/j.tplants.2007.04.001

Pourrut, B., Shahid, M., Douay, F., Dumat, C., and Pinelli, E. (2013). "Molecular mechanisms involved in lead uptake, toxicity and detoxification in higher plants," in Heavy Metal Stress in Plants, eds D. K. Gupta, F. J. Corpas, and J. M. Palma (Berlin; Heidelberg: Springer-Verlag), 121-147.

Qiao, J., Wang, J., Chen, L., Tian, X., Huang, S., Ren, X., et al. (2012). Quantitative iTRAQ LC-MS/MS proteomics reveals metabolic responses to biofuel ethanol in cyanobacterial Synechocystis sp. PCC 6803. J. Proteome Res. 11, 5286-5300. doi: $10.1021 / p r 300504 \mathrm{w}$

Ralhan, R., DeSouza, L. V., Matta, A., Tripathi, S. C., Ghanny, S., Gupta, S. D., et al. (2008). Discovery and verification of head-and-neck cancer biomarkers by differential protein expression analysis using iTRAQ labeling, multidimensional liquid chromatography, and tandem mass spectrometry. Mol. Cell. Proteomics 7, 1162-1173. doi: 10.1074/mcp.M700500-MCP200

Saravanan, R. S., and Rose, J. K. (2004). A critical evaluation of sample extraction techniques for enhanced proteomic analysis of recalcitrant plant tissues. Proteomics 4, 2522-2532. doi: 10.1002/pmic.200300789

Sarry, J. E., Kuhn, L., Ducruix, C., Lafaye, A., Junot, C., Hugouvieux, V., et al. (2006). The early responses of Arabidopsis thaliana cells to cadmium exposure explored by protein and metabolite profiling analyses. Proteomics 6, 2180-2198. doi: $10.1002 /$ pmic. 200500543

Sazuka, T., Keta, S., Shiratake, K., Yamaki, S., and Shibata, D. (2004). A proteomic approach to identification of transmembrane proteins and membraneanchored proteins of Arabidopsis thaliana by peptide sequencing. DNA Res. 11, 101-113. doi: 10.1093/dnares/11.2.101

Schommer, C., Palatnik, J. F., Aggarwal, P., Chételat, A., Cubas, P., Farmer, E. E., et al. (2008). Control of jasmonate biosynthesis and senescence by miR319 targets. PLoS Biol. 6:e230. doi: 10.1371/journal.pbio.0060230

Sebastiani, L., Francini, A., Romeo, S., Ariani, A., and Minnocci, A. (2014). "Heavy metals stress on poplar: molecular and anatomical modifications," in Approaches to Plant Stress and Their Management, eds R. K. Gaur and P. Sharma (New Delhi: Springer), 267-279.

Sharma, P., and Dubey, R. S. (2005). Lead toxicity in plants. Braz. J. Plant Physiol. 17, 35-52. doi: 10.1590/S1677-04202005000100004

Singh, S., Parihar, P., Singh, R., Prasad, S. M., and Singh, V. P. (2016). Heavy metal tolerance in plants: role of transcriptomics, proteomics, metabolomics, and ionomics. Front. Plant Sci. 6:1143. doi: 10.3389/fpls.2015.01143

Srivastava, S., Srivastava, A. K., Suprasanna, P., and D'Souza, S. F. (2009). Comparative biochemical and transcriptional profiling of two contrasting varieties of Brassica juncea L. in response to arsenic exposure reveals mechanisms of stress perception and tolerance. J. Exp. Bot. 60, 3419-3431. doi: 10.1093/jxb/erp181

Suzuki, N., Koussevitzky, S., Mittler, R., and Miller, G. (2012). ROS and redox signalling in the response of plants to abiotic stress. Plant Cell Environ. 35, 259-270. doi: 10.1111/j.1365-3040.2011.02336.x

Thapa, G., Sadhukhan, A., Panda, S. K., and Sahoo, L. (2012). Molecular mechanistic model of plant heavy metal tolerance. Biometals 25, 489-505. doi: 10.1007/s10534-012-9541-y

Vuoristo, K. S., Mars, A. E., Sanders, J. P., Eggink, G., and Weusthuis, R. A. (2015). Metabolic engineering of TCA cycle for production of chemicals. Trends Biotechnol. 34, 191-197. doi: 10.1016/j.tibtech.2015.11.002

Wang, C. Y., Shen, R. F., Wang, C., and Wang, W. (2013). Root protein profile changes induced by $\mathrm{Al}$ exposure in two rice cultivars differing in $\mathrm{Al}$ tolerance. J. Proteomics 78, 281-293. doi: 10.1016/j.jprot.2012.09.035

Wang, L., and He, Q. (2005). China Radish. Beijing: Scientific and Technical Documents Publishing House Press. 
Wang, Y., Shen, H., Xu, L., Zhu, X. W., Li, C., Zhang, W., et al. (2015a). Transport, ultrastructural localization, and distribution of chemical forms of lead in radish (Raphanus sativus L.). Front. Plant Sci. 6:293. doi: 10.3389/fpls.2015.00293

Wang, Y., Xu, L., Shen, H., Wang, J., Liu, W., Zhu, X., et al. (2015b). Metabolomic analysis with GC-MS to reveal potential metabolites and biological pathways involved in $\mathrm{Pb} \& \mathrm{Cd}$ stress response of radish roots. Sci. Rep. 5:18296. doi: $10.1038 /$ srep 18296

Wang, Z. Q., Xu, X. Y., Gong, Q. Q., Xie, C., Fan, W., Yang, J. L., et al. (2014). Root proteome of rice studied by iTRAQ provides integrated insight into aluminum stress tolerance mechanisms in plants. J. Proteomics 98, 189-205. doi: 10.1016/j.jprot.2013.12.023

Xie, Y., Ye, S., Wang, Y., Xu, L., Zhu, X. W., Yang, J. L., et al. (2015). Transcriptomebased gene profiling provides novel insights into the characteristics of radish root response to $\mathrm{Cr}$ stress with next-generation sequencing. Front. Plant Sci. 6:202. doi: 10.3389/fpls.2015.00202

Xu, L., Wang, L., Gong, Y., Dai, W., Wang, Y., Zhu, X., et al. (2012). Genetic linkage map construction and QTL mapping of cadmium accumulation in radish (Raphanus sativus L.). Theor. Appl. Genet. 125, 659-670. doi: 10.1007/s00122-012-1858-y

Yang, L., Jiang, T. B., Fountain, J. C., Scully, B. T., Lee, R. D., Kemerait, R. C., et al. (2014). Protein profiles reveal diverse responsive signaling pathways in kernels of two maize inbred lines with contrasting drought sensitivity. Int. J. Mol. Sci. 15, 18892-18918. doi: 10.3390/ijms151018892

Yang, L. T., Qi, Y. P., Lu, Y. B., Guo, P., Sang, W., Feng, H., et al. (2013). iTRAQ protein profile analysis of Citrus sinensis roots in response to long-term boron-deficiency. J. Proteomics 93, 179-206. doi: 10.1016/j.jprot.2013.04.025

Zhuang, W., Gao, Z., Wang, L., Zhong, W., Ni, Z., and Zhang, Z. (2013). Comparative proteomic and transcriptomic approaches to address the active role of GA4 in Japanese apricot flower bud dormancy release. J. Exp. Bot. 64, 4953-4966. doi: 10.1093/jxb/ert284

Conflict of Interest Statement: The authors declare that the research was conducted in the absence of any commercial or financial relationships that could be construed as a potential conflict of interest.

Copyright (C) 2016 Wang, Xu, Tang, Jiang, Chen, Zhang, Wang and Liu. This is an open-access article distributed under the terms of the Creative Commons Attribution License (CC BY). The use, distribution or reproduction in other forums is permitted, provided the original author(s) or licensor are credited and that the original publication in this journal is cited, in accordance with accepted academic practice. No use, distribution or reproduction is permitted which does not comply with these terms. 